\title{
Seed Morphology-Directed Synthesis of Concave Gold Nanocrystals with Tunable Sizes
}

Gongguo Zhang, ${ }^{a}$ Yanyun Ma, ${ }^{\mathrm{b}}$ Zhiang Liu, ${ }^{\mathrm{c}}$ Xiaowei Fu, ${ }^{\mathrm{a}}$ Xiankang Niu, ${ }^{\mathrm{c}}$ Fengli Qu, ${ }^{\mathrm{c}}$ Chongdian

$\mathrm{Si}^{\mathrm{a}}$ and Yiqun Zheng ${ }^{* \mathrm{a}}$

Number of pages: 26

Number of figures: 18

Number of schemes:0

Number of tables: 3

\section{Table of Content}

\section{Details of electrochemical measurements}

Figure S1. UV-vis extinction spectra and peak positions recorded as different amounts of Au precursor was added in growth solution.

Figure S2. Histogram showing the size distribution of concave Au nanocubes.

Figure S3. TEM images showing the time-lapse study of growth of concave Au nanocubes.

Figure S4. XRD pattern of concave Au nanocubes. 
Figure S5. EDS spectrum of concave Au nanocubes.

Figure S6. HRTEM image of an individual concave Au nanocube.

Figure S7. Three-dimensional models of concave cubes.

Figure S8. SEM images showing the effect of capping agent on product morphology.

Figure S9. TEM images of spherical Au seeds with an average diameter of a) $20 \mathrm{~nm}$ and b) $40 \mathrm{~nm}$.

Figure S10. SEM images showing the effect of seed size on product morphology.

Figure S11. Histograms showing the size distribution for concave Au nanocubes.

Figure S12. TEM image of rod-like Au seeds.

Figure S13. Histograms showing the size distribution for concave Au nanobars.

Figure S14. SEM image of rod-like products obtained via the standard procedure, except for the use of rod-like seeds with different volumes.

Figure S15. UV-vis extinction spectra of rod-like Au products as displayed in Figure S14.

Figure S16. SEM image of 40-nm, octahedral Au nanoparticles.

Figure S17. TEM images of concave nanocube and nanobars with dictated projection angles and corresponding geometric models.

Figure S18. HRTEM images showing the step surface of concave nanocube and nanobar.

Table S1. Summary of recent progress in controlled synthesis of concave Au nanocrystals.

Table S2. Synthetic parameters for concave Au nanocrystals with different sizes and shapes.

Table S3. Size information of samples displayed in current work. 


\section{Details of electrochemical measurements}

The as-prepared $\mathrm{Au}$ nanocrystals were washed twice by centrifugation and $3 \mu \mathrm{L}$ of the concentrated suspension was dropped onto surface of glassy carbon electrode (GCE) and dried naturally, followed by coating using $3 \mu \mathrm{L}$ of Nafion solution and dried naturally. The Au-modified

GCE was then used as the working electrode. The $\mathrm{Ag} / \mathrm{AgCl}$ electrode and Pt wire was employed as the counter and reference electrodes, respectively. An aqueous solution of $\mathrm{H}_{2} \mathrm{SO}_{4}(0.1 \mathrm{M})$, purged with high-purity $\mathrm{N}_{2}$ gas for 30 min prior to measurements, was used as the electrolyte solution. The scan rate of voltage was set to $10 \mathrm{mV} / \mathrm{s}$. The first two cycles of oxidation and reduction were discarded and the data recorded during the third cycle were displayed in main text, where the oxidation and reduction traces were normalized against the maximum reduction current for each sample. 

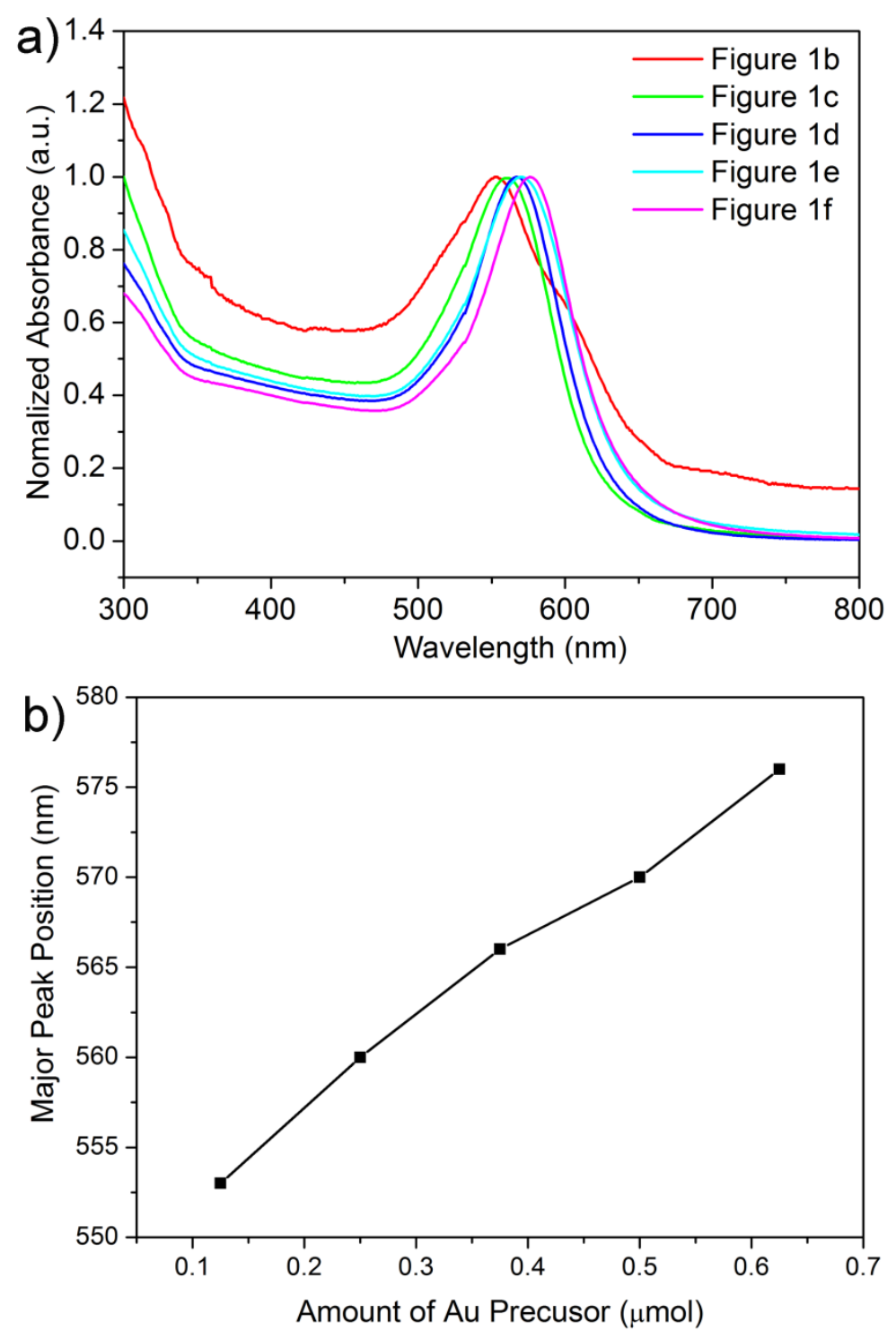

Figure S1. a) Normalized UV-vis extinction spectra recorded as different amounts of Au precursor was added in growth solution. b) Plot showing the relationship between amount of Au precursor and major peak position. 


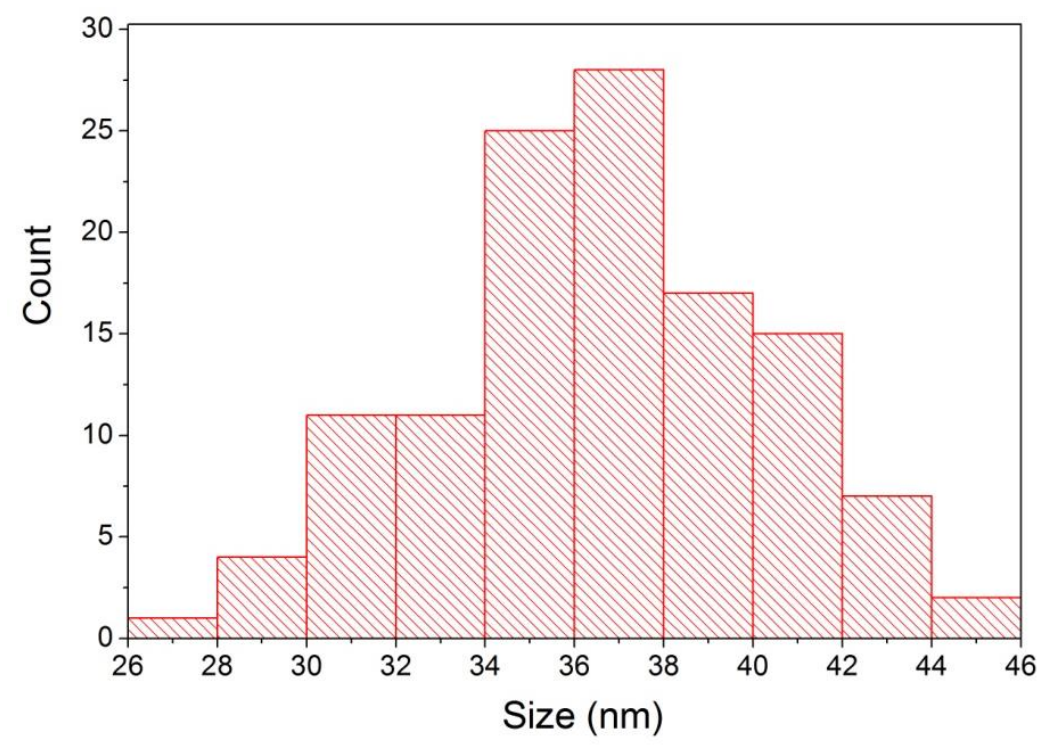

Figure S2. Histogram showing the size distribution of concave Au nanocubes as displayed in Figure 2 . They were statistically measured by counting over 100 typical particles in corresponding SEM images. 

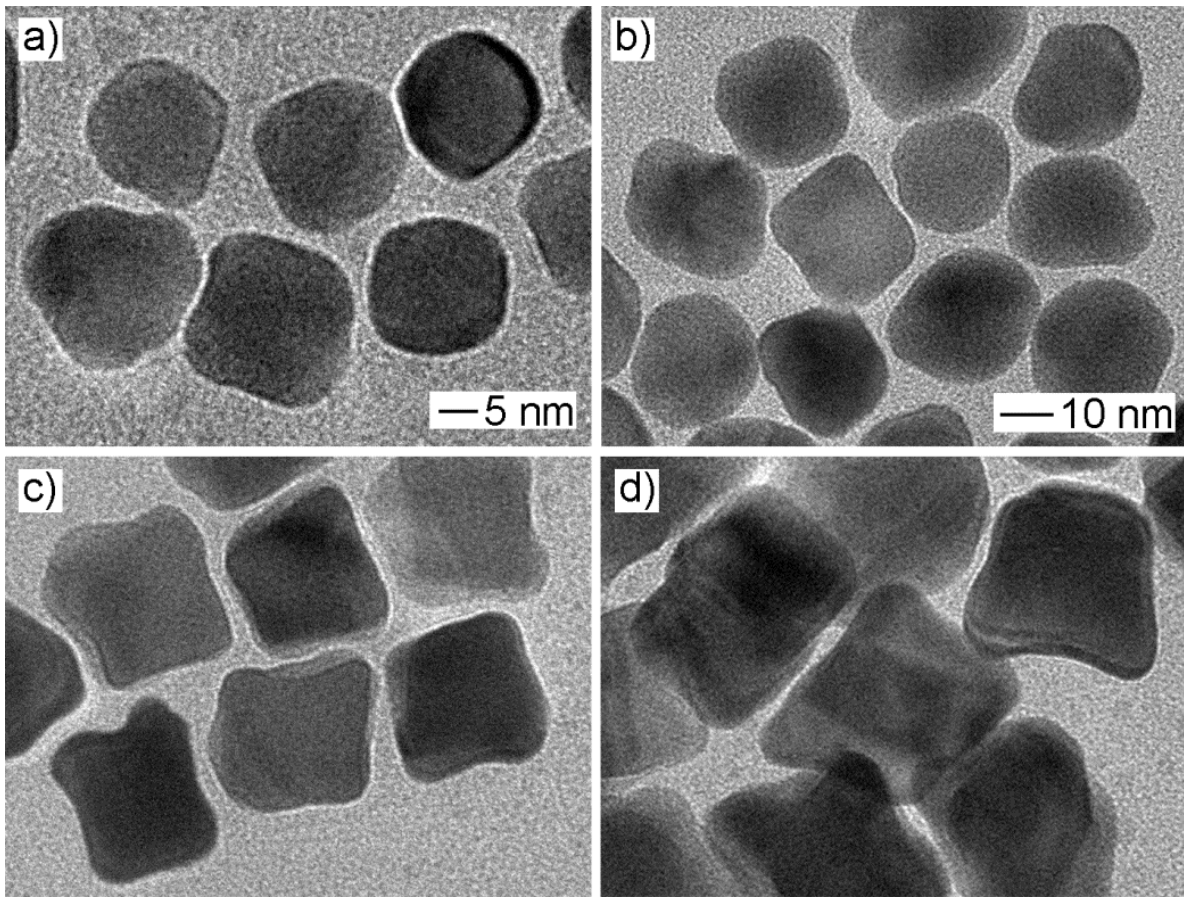

Figure S3. Time-lapse study of growth process of concave Au nanocubes. TEM images of $\mathrm{Au}$ products taken from the reaction mixture conducted via the standard procedure for different periods: a) $5 \mathrm{~min}$; b) $20 \mathrm{~min}$; c) $40 \mathrm{~min}$; d) $60 \mathrm{~min}$, respectively. The scale bar in b) applies to c) and d). 


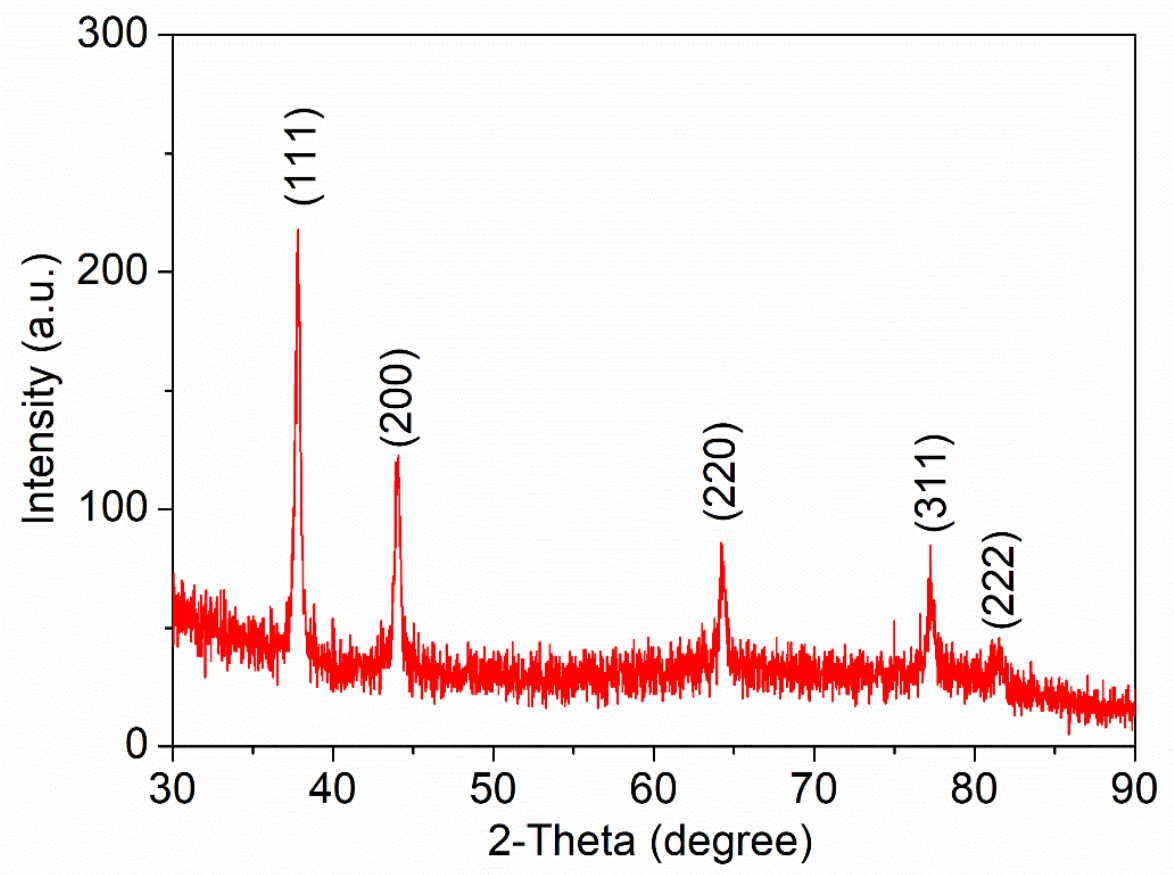

Figure S4. XRD pattern of concave Au nanocubes as displayed in Figure 2. 


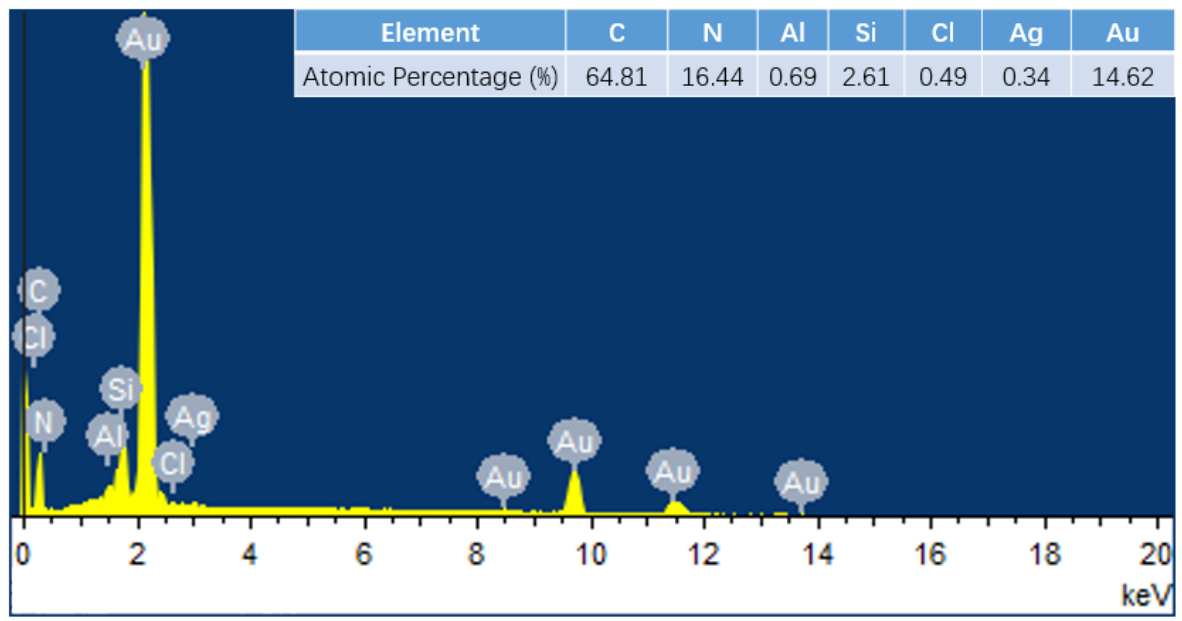

Figure S5. EDS spectrum of concave Au nanocubes as displayed in Figure 2. 


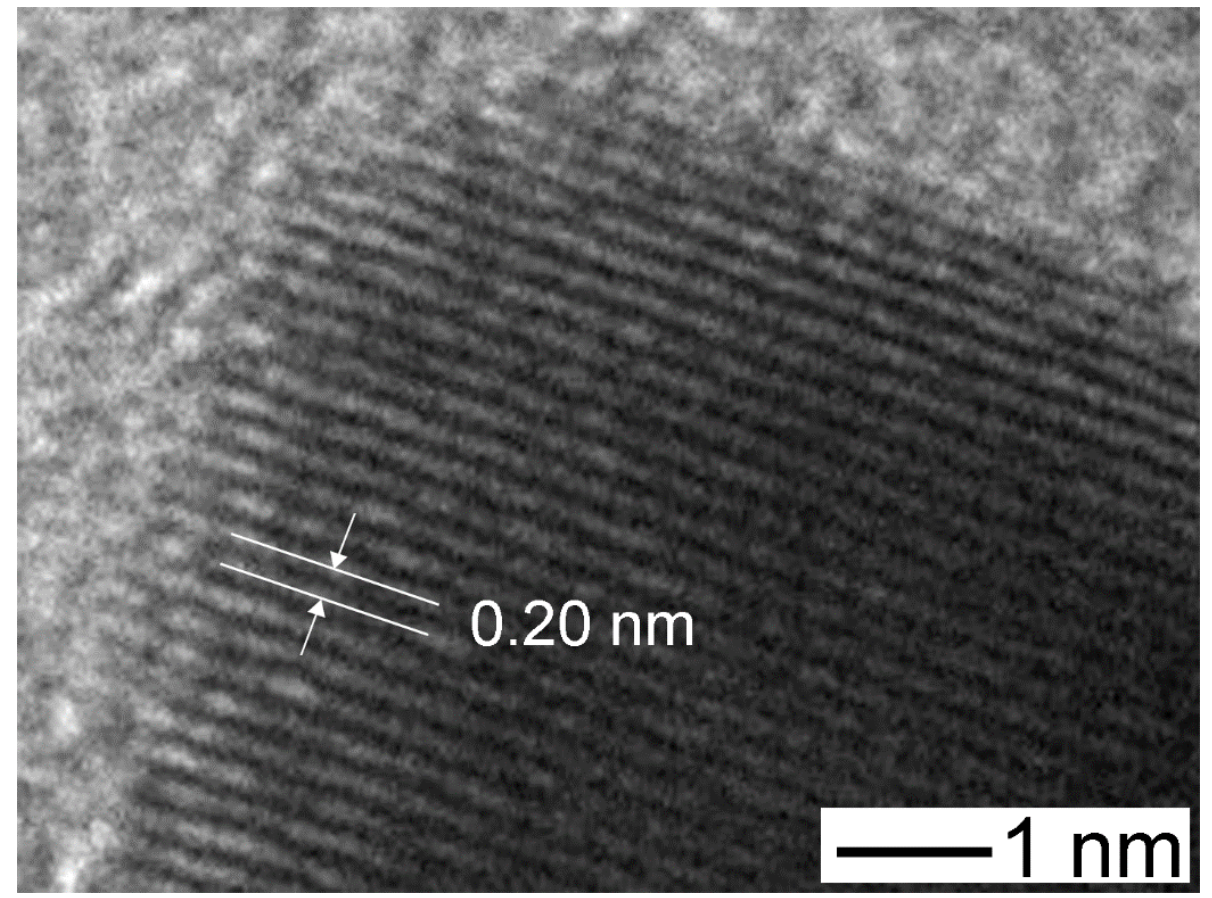

Figure S6. HRTEM image of the corner region of a concave cubic Au nanoparticle. 
[100]
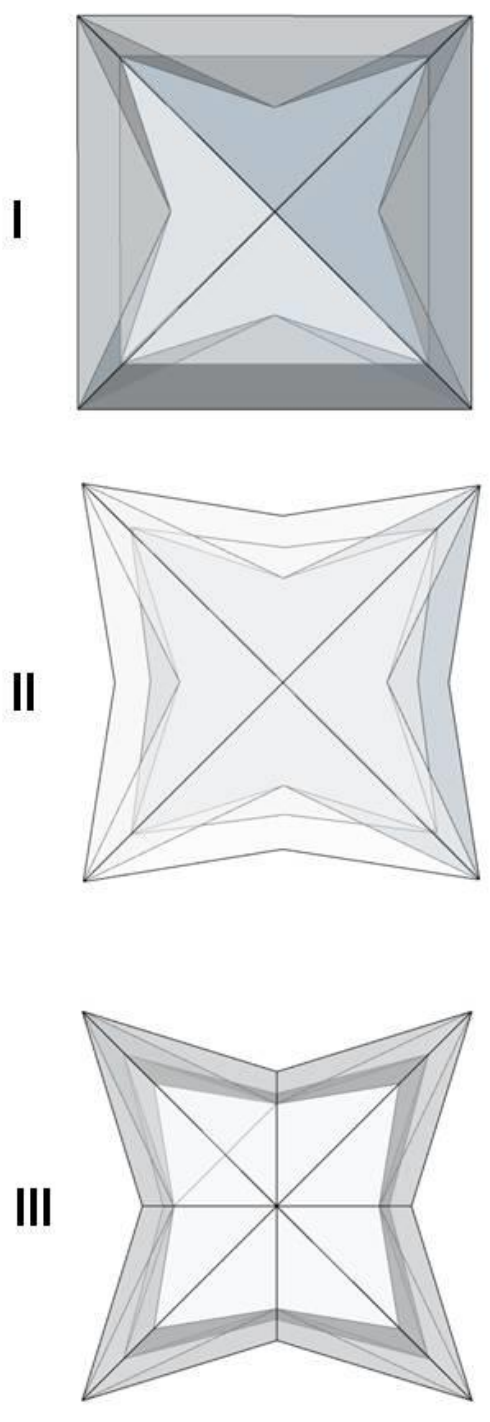

[110]
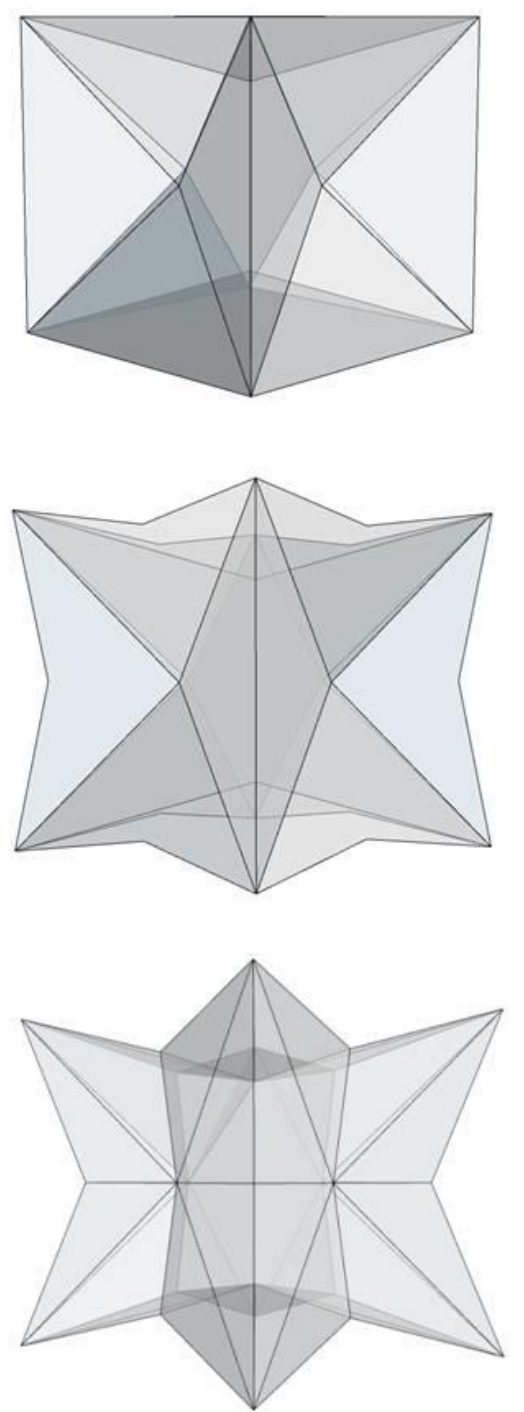

[111]
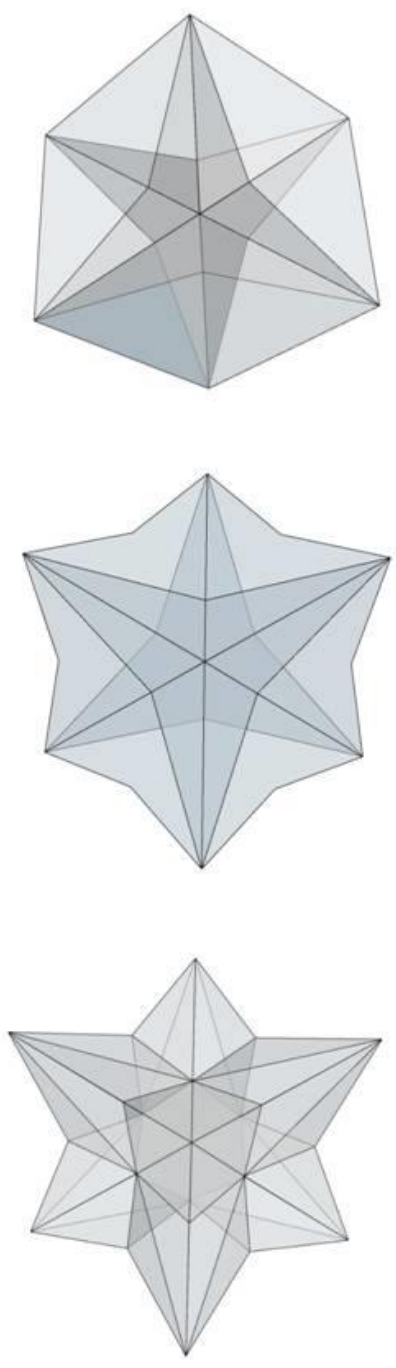

Figure S7. Three-dimensional models of concave cubes with straight edge (Model I) and curved edges with different degrees (Model II \& III) viewed along [100], [110], and [111] directions. 

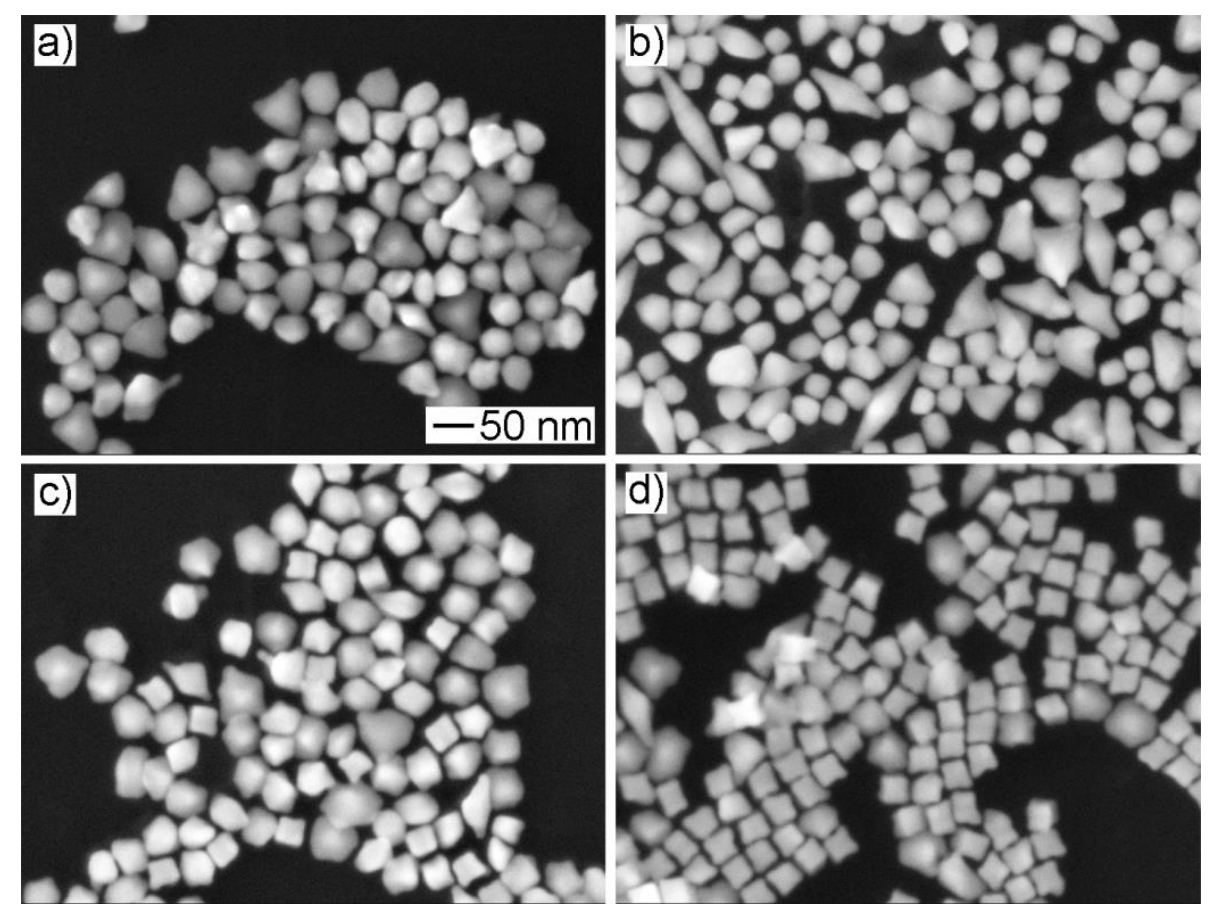

Figure S8. Effect of capping agent on product morphology. SEM images of products obtained via the standard procedure, except that the capping agent combination were varied from CTAC (75 $\mathrm{mM})$ \& CTAB $(25 \mathrm{mM})$ in standard procedure to a) CTAC $(100 \mathrm{mM})$, b) CTAB $(100 \mathrm{mM})$, c) CTAC (87.5 mM) \& CTAB (12.5 mM), and d) CTAC $(62.5 \mathrm{mM}) \&$ CTAB $(37.5 \mathrm{mM})$, respectively. The scale bar in a) applies to b-d). 

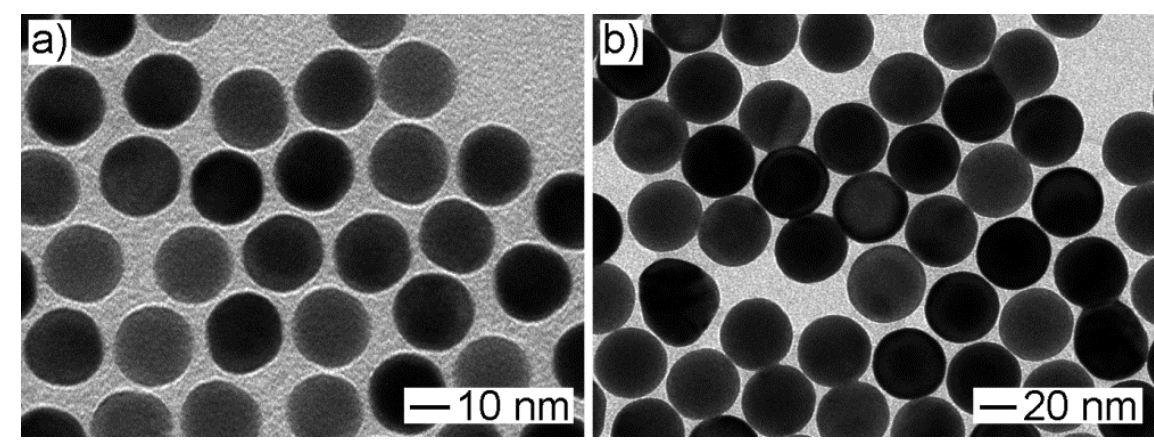

Figure S9. TEM images of spherical Au seeds with an average diameter of a) $20 \mathrm{~nm}$ and b) $40 \mathrm{~nm}$. 

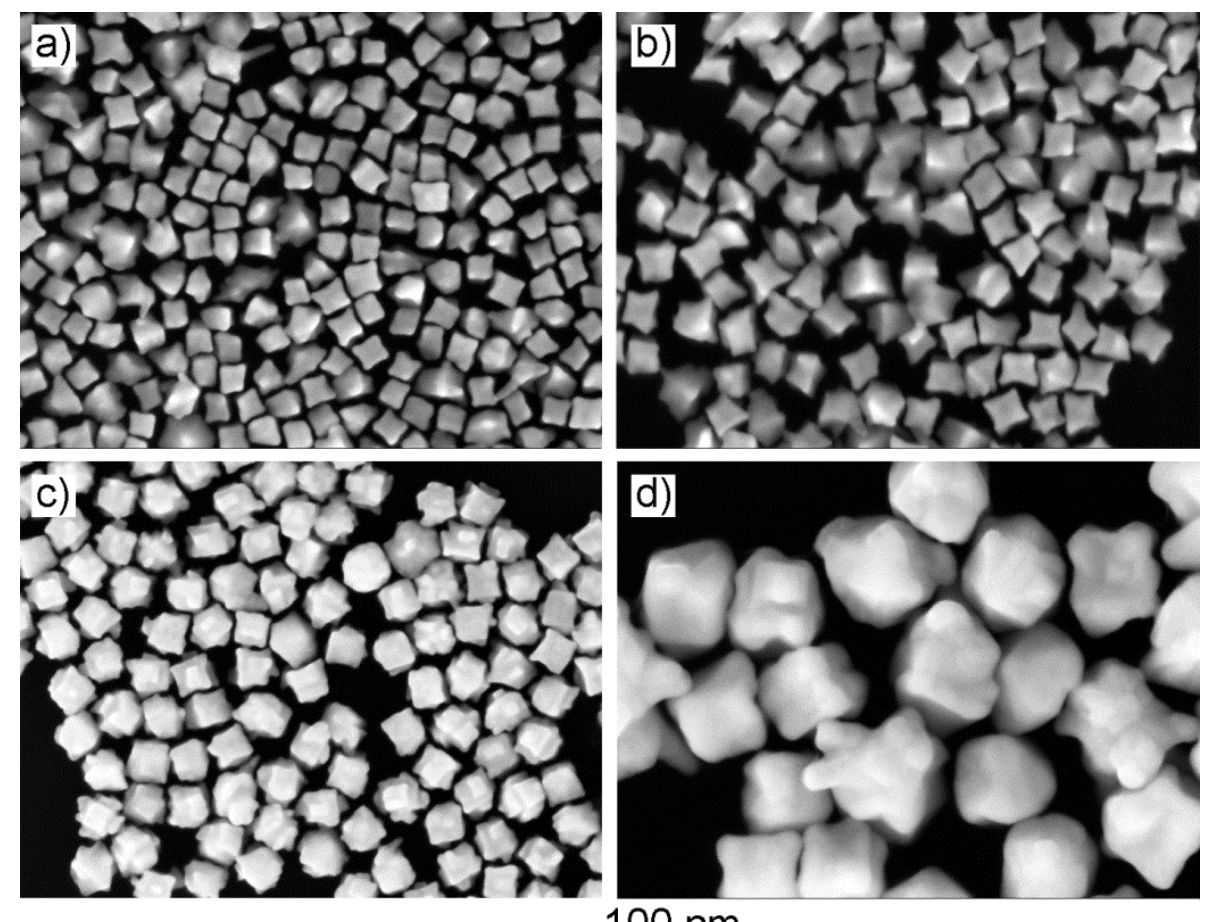

$-100 \mathrm{~nm}$

Figure S10. Effect of seed size on product morphology. SEM image of Au products obtained via the standard procedure, except that the $10-\mathrm{nm}$, spherical Au seeds (20 $\mu \mathrm{L})$ was replaced by: a, b) 20-nm, spherical Au seeds with the volume of a) 100 and b) $50 \mu \mathrm{L}$, respectively; c, d) 40-nm, spherical Au seeds with the volume of c) 100 and d) $10 \mu \mathrm{L}$, respectively. 

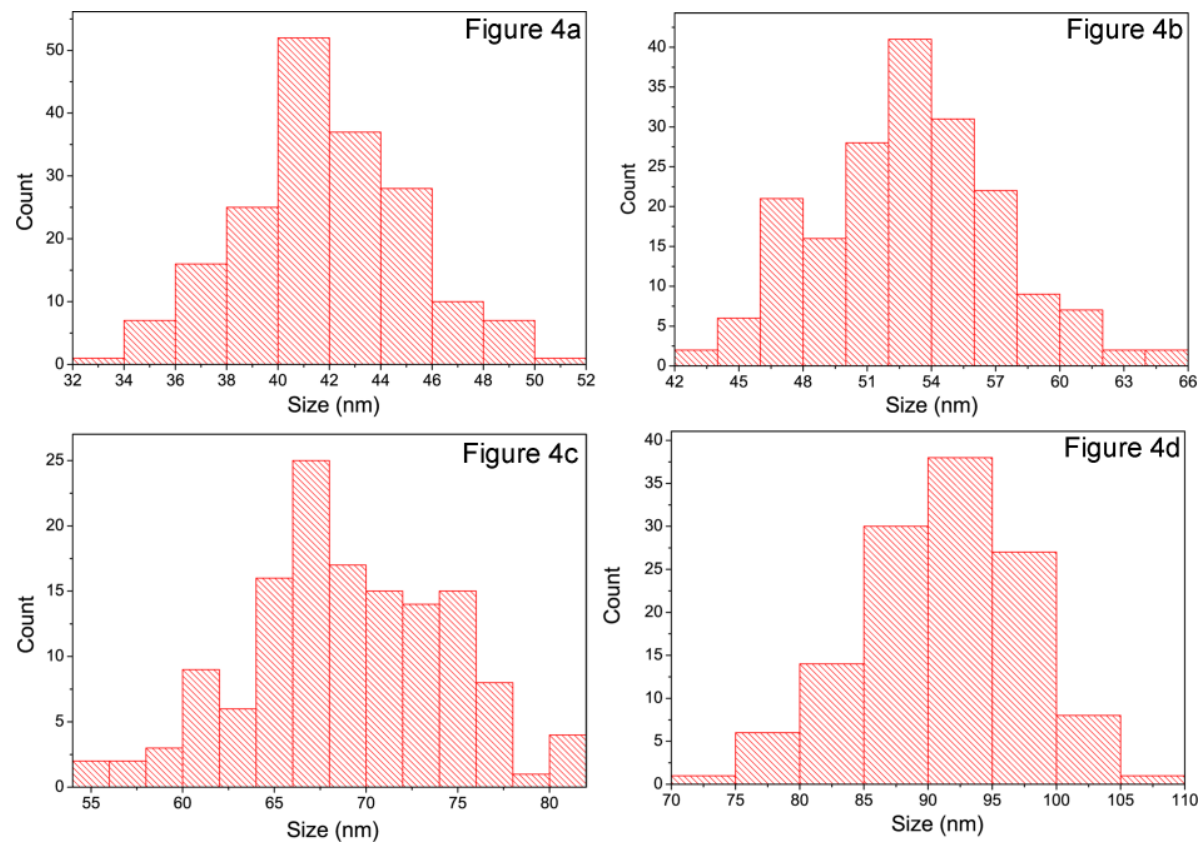

Figure S11. Histograms showing the size distribution for concave Au nanocubes as displayed in

Figure 4. They were statistically measured by counting over 100 typical particles in corresponding

SEM images. 


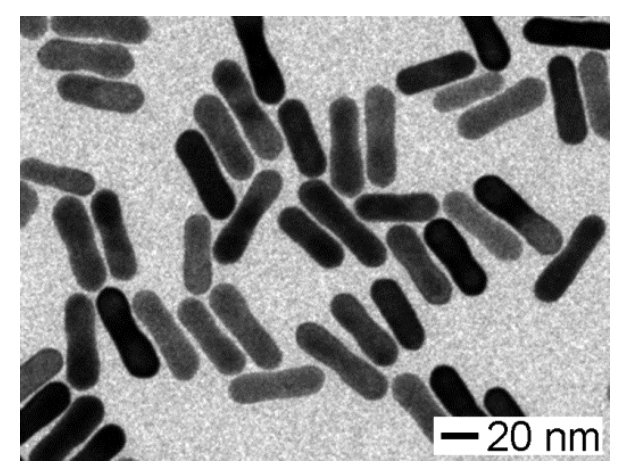

Figure S12. TEM image of rod-like Au seeds. 

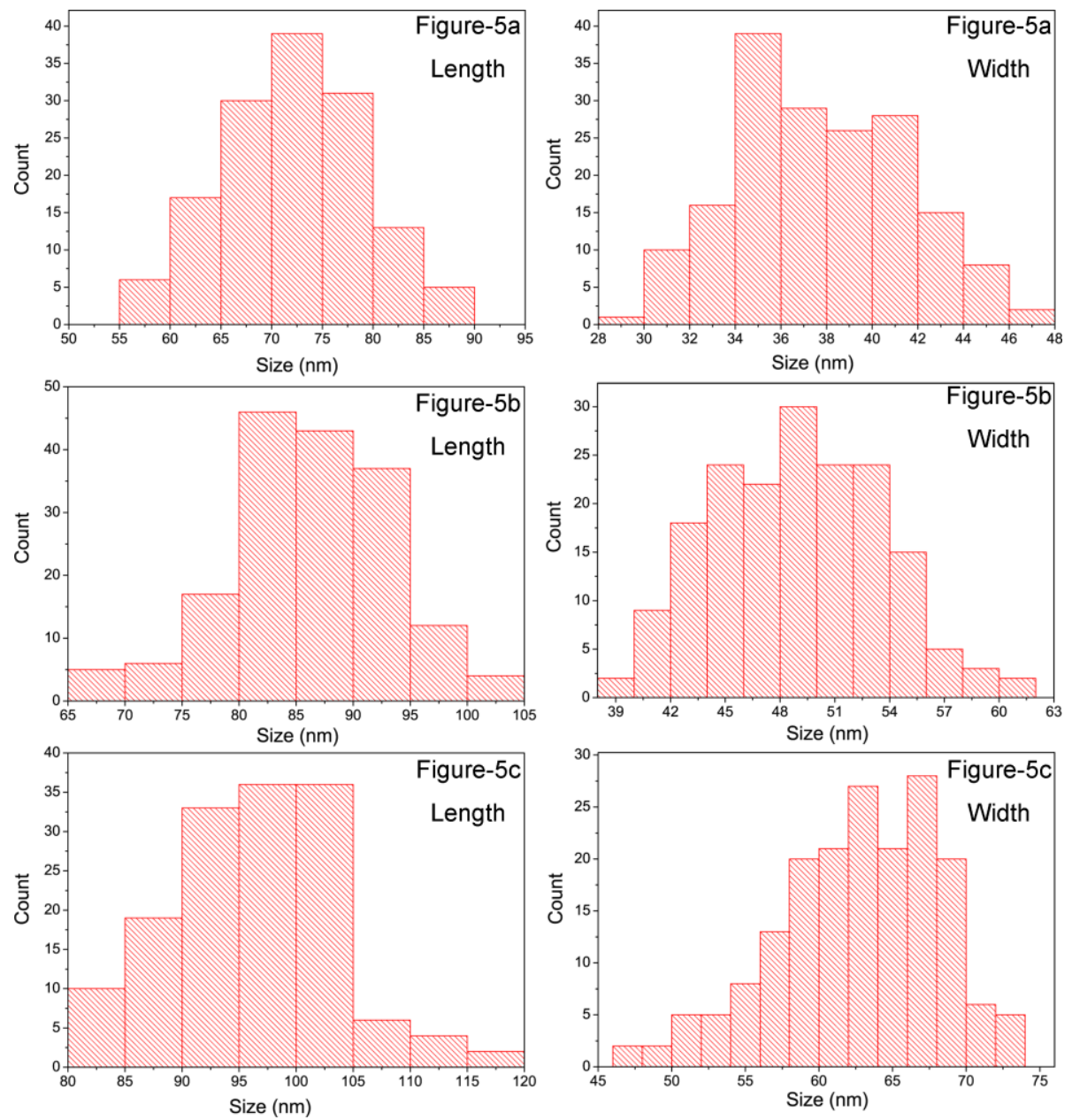

Figure S13. Histograms showing the size distribution for concave Au nanobars as displayed in

Figure 5. They were statistically measured by counting over 100 typical particles in corresponding

TEM images. 


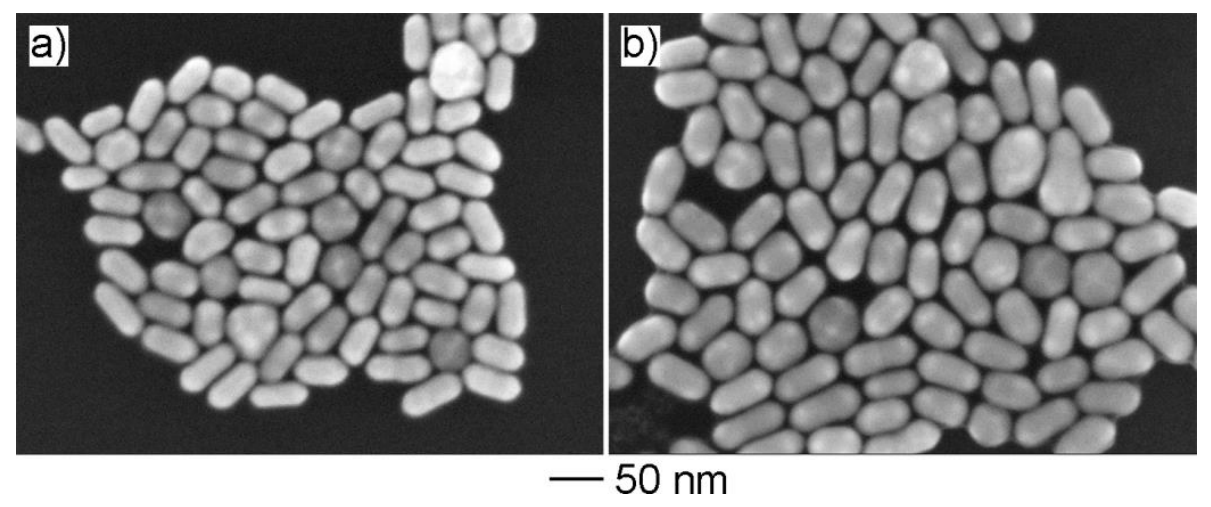

Figure S14. SEM image of rod-like products obtained via the standard procedure, except that the spherical Au seeds were replaced by rod-like ones with the volume of a) 200 and b) $100 \mu \mathrm{L}$, respectively. 


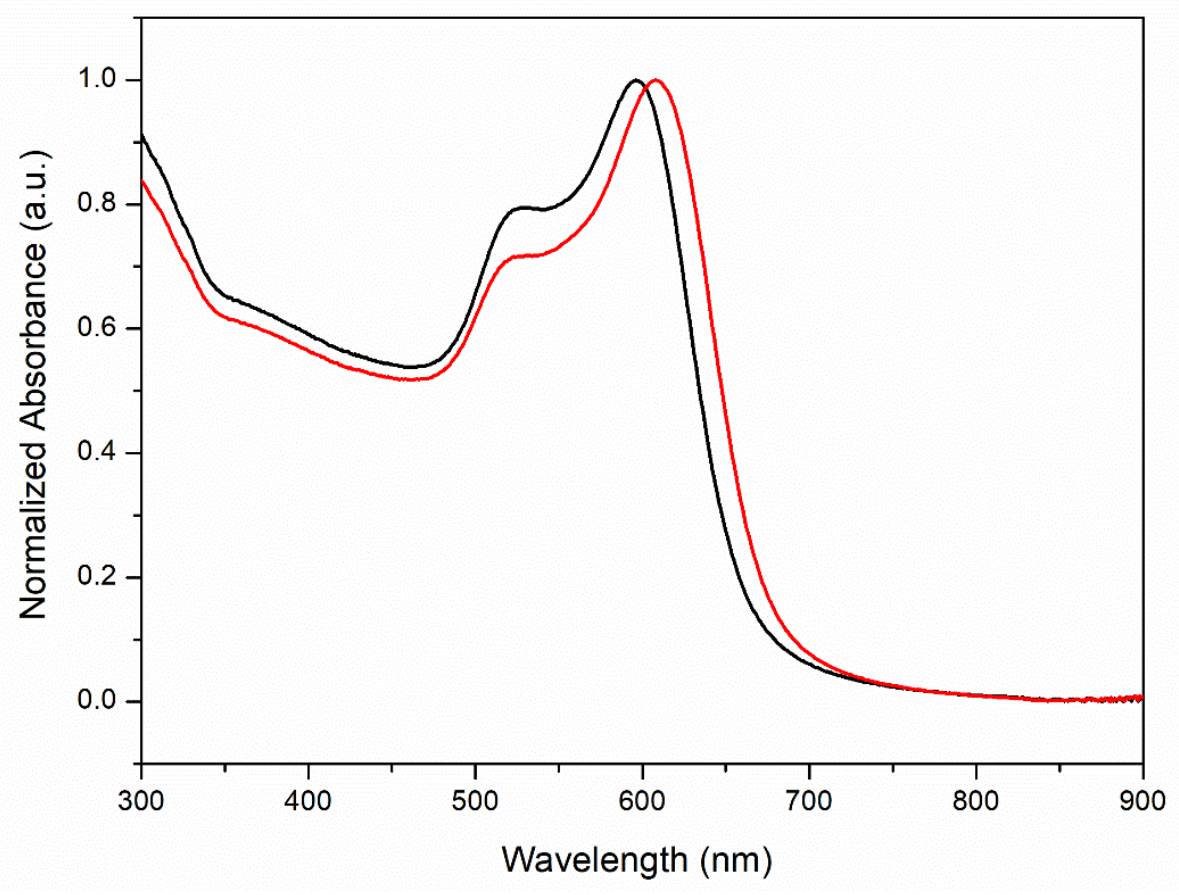

Figure S15. UV-vis extinction spectra of rod-like Au products as displayed in Figure S14. The black line corresponds to the sample shown in Figure S14a and the red line corresponds to the sample shown in Figure S14b. 


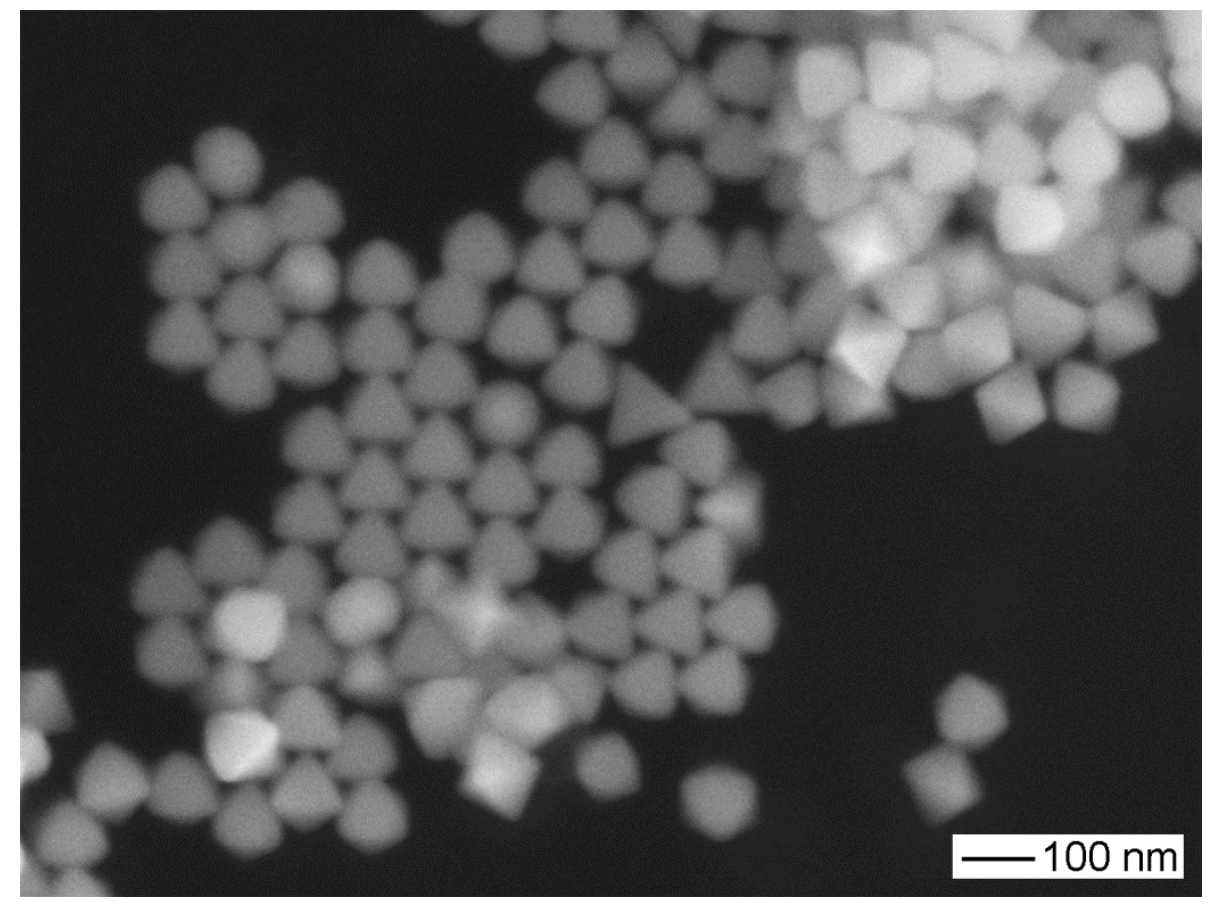

Figure S16. SEM image of 40-nm, octahedral Au nanoparticles. 

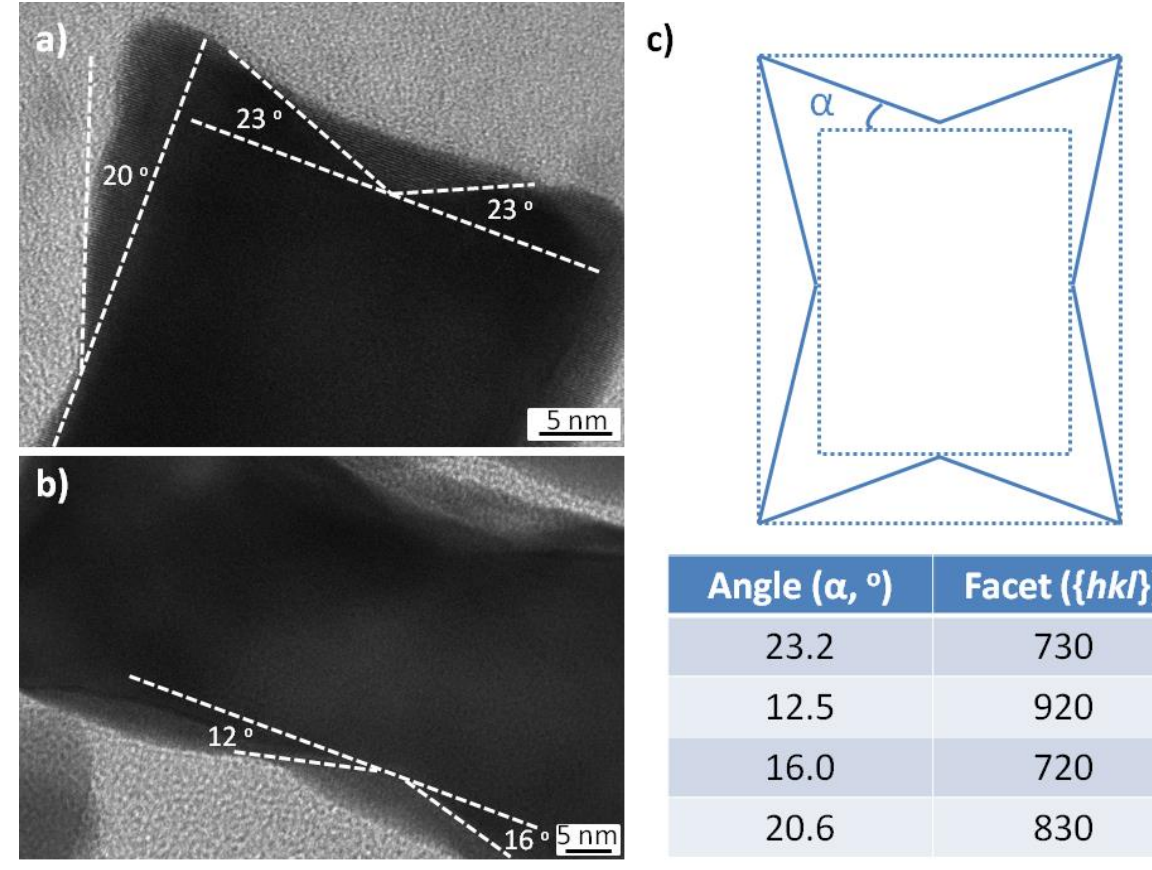

\begin{tabular}{|c|c|}
\hline Angle $\left(\boldsymbol{\alpha},{ }^{\circ}\right)$ & Facet $(\{h k /\})$ \\
\hline 23.2 & 730 \\
\hline 12.5 & 920 \\
\hline 16.0 & 720 \\
\hline 20.6 & 830 \\
\hline
\end{tabular}

Figure S17. a, b) TEM images of an individual Au particle in the form of concave a) cube and b) bar. The dotted lines were plotted to dictate the projection angle. c) Corresponding geometric model and table illustrating the relationship between projection angle and exposed facets. 

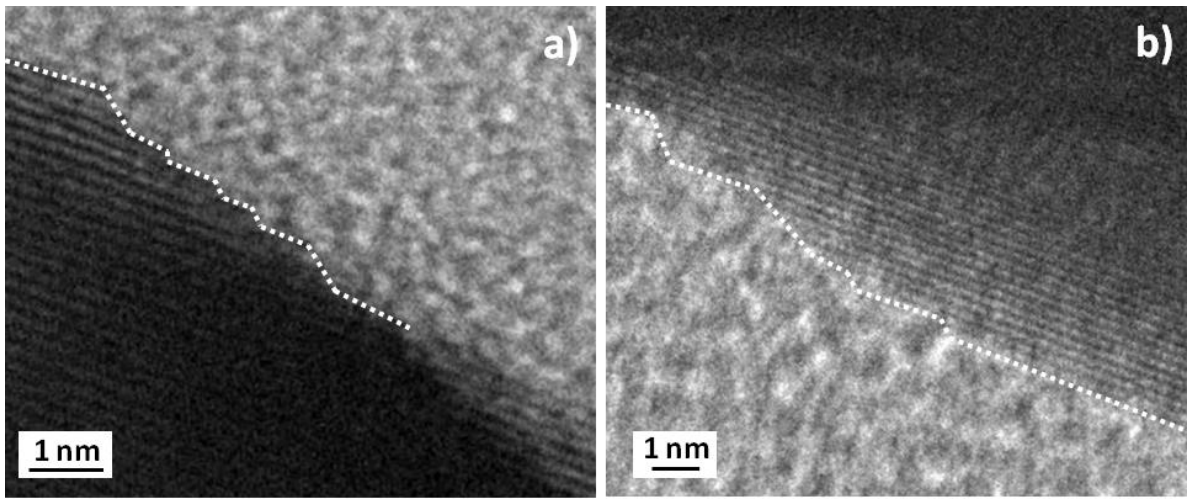

Figure S18. HRTEM images showing the step surface of concave a) nanocube and b) nanobar. 
Table S1. Summary of recent progress in controlled synthesis of concave Au nanocrystals.

\begin{tabular}{|c|c|c|c|c|c|}
\hline No. & Shape & Seed & $\begin{array}{c}\text { Reagents in Growth } \\
\text { Solution }\end{array}$ & Tunable Size Range & Ref. \\
\hline 1 & $\begin{array}{l}\text { concave cube } \\
\text { (tetrapod) }\end{array}$ & CTAB-Au seeds ${ }^{\mathrm{a}}$ & CTAB $/ \mathrm{Ag}^{+} / \mathrm{AA}$ & $\mathrm{n} / \mathrm{a}$ & 1 \\
\hline 2 & concave cube & CTAC-Au seeds ${ }^{\mathrm{a}}$ & $\mathrm{CTAC} / \mathrm{Ag}^{+} / \mathrm{HCl} / \mathrm{AA}$ & $38 \sim 269 \mathrm{~nm}$ & 2 \\
\hline 3 & concave plate & Au nanoplates & PDDA & $\mathrm{n} / \mathrm{a}$ & 3 \\
\hline 4 & $\begin{array}{l}\text { concave } \\
\text { bipyramid }\end{array}$ & Au bipyramids & $\mathrm{CTBAB}, \mathrm{Ag}+, \mathrm{AA}$ & $\begin{array}{l}\text { Length: } 119 \sim 560 \mathrm{~nm} \text {; } \\
\text { Width: } 42 \sim 99 \mathrm{~nm}\end{array}$ & 4 \\
\hline 5 & concave nanobar & Au nanrods & $\mathrm{CTAB} / \mathrm{Ag}^{+} / \mathrm{HCl} / \mathrm{AA}$ & $\mathrm{n} / \mathrm{a}$ & 5 \\
\hline 6 & concave cuboid & Au nanrods & CTAB, AA & $\begin{array}{l}\text { Length: } 70 \sim 140 \mathrm{~nm} ; \\
\text { Width: } 40 \sim 68 \mathrm{~nm}\end{array}$ & 6 \\
\hline 7 & concave nanobar & Au nanrods & $\mathrm{DDAB} / \mathrm{Ag}^{+} / \mathrm{AA}$ & $\mathrm{n} / \mathrm{a}$ & 7 \\
\hline 8 & concave cuboid & Au nanrods & $\mathrm{Cu}^{2+} / \mathrm{CTAB} / \mathrm{AA}$ & $\mathrm{n} / \mathrm{a}$ & $8-9$ \\
\hline 9 & concave cuboid & Au nanrods & $\begin{array}{c}\text { tannic acid/CTAC/ } \\
\mathrm{Ag}^{+} / \mathrm{NaOH}\end{array}$ & $\sim 80$ to $\sim 200 \mathrm{~nm}$ & 10 \\
\hline 10 & dog bone & Au nanrods & $\begin{array}{c}\mathrm{CTAB} / \mathrm{Ag}^{+} / \mathrm{HCl}(\mathrm{NaOH}) / \\
\text { hydroquinone }\end{array}$ & $\mathrm{n} / \mathrm{a}$ & 11 \\
\hline \multirow{2}{*}{$\begin{array}{c}\text { Current } \\
\text { work }\end{array}$} & concave cube & Au nanospheres & \multirow[b]{2}{*}{ CTAC/CTAB $/ \mathrm{Ag}^{+} / \mathrm{AA}$} & $37 \sim 91 \mathrm{~nm}$ & I \\
\hline & concave bar & Au nanorods & & $\begin{array}{l}\text { Length: 72 96 nm; } \\
\text { Width: 38 63 nm }\end{array}$ & / \\
\hline
\end{tabular}

${ }^{\text {a }} \mathrm{CTAX}(\mathrm{X}=\mathrm{Cl}, \mathrm{Br})$-Au seeds were obtained by reacting mixture of $\mathrm{HAuCl}_{4}$ and $\mathrm{CTAX}(\mathrm{X}=\mathrm{Cl}, \mathrm{Br})$ with $\mathrm{NaBH}_{4}$. 
Table S2. Synthetic parameters for concave Au nanocrystals with different sizes and shapes.

\begin{tabular}{|c|c|c|c|c|c|c|c|c|}
\hline Sample & $\begin{array}{c}\text { CTAC } \\
(200 \mathrm{mM})\end{array}$ & $\begin{array}{c}\text { CTAB } \\
(200 \mathrm{mM})\end{array}$ & $\begin{array}{l}\mathrm{AgNO}_{3} \\
(2 \mathrm{mM})\end{array}$ & $\begin{array}{c}\mathrm{AA} \\
(10 \mathrm{mM})\end{array}$ & $\begin{array}{c}\text { Spherical } \\
\text { Seeds }\end{array}$ & $\begin{array}{c}\text { Rod-like } \\
\text { Seeds }\end{array}$ & $\begin{array}{c}\mathrm{HAuCl}_{4} \\
(0.5 \mathrm{mM})\end{array}$ & $\mathrm{H}_{2} \mathrm{O}$ \\
\hline $\begin{array}{c}\text { CGC } \\
(37 \mathrm{~nm})\end{array}$ & $1.5 \mathrm{~mL}$ & $0.5 \mathrm{~mL}$ & $10 \mu \mathrm{L}$ & $110 \mu \mathrm{L}$ & $20 \mu \mathrm{L}$ & I & $1.5 \mathrm{~mL}$ & $0.36 \mathrm{~mL}$ \\
\hline $\begin{array}{c}\text { CGC } \\
\text { (42 nm) }\end{array}$ & $1.5 \mathrm{~mL}$ & $0.5 \mathrm{~mL}$ & $10 \mu \mathrm{L}$ & $110 \mu \mathrm{L}$ & $10 \mu \mathrm{L}$ & I & $1.5 \mathrm{~mL}$ & $0.37 \mathrm{~mL}$ \\
\hline $\begin{array}{c}\text { CGC } \\
(53 \mathrm{~nm})\end{array}$ & $1.5 \mathrm{~mL}$ & $0.5 \mathrm{~mL}$ & $10 \mu \mathrm{L}$ & $110 \mu \mathrm{L}$ & $5 \mu \mathrm{L}$ & I & $1.5 \mathrm{~mL}$ & $0.38 \mathrm{~mL}$ \\
\hline $\begin{array}{c}\text { CGC } \\
(69 \mathrm{~nm})\end{array}$ & $1.5 \mathrm{~mL}$ & $0.5 \mathrm{~mL}$ & $10 \mu \mathrm{L}$ & $110 \mu \mathrm{L}$ & $2 \mu \mathrm{L}$ & I & $1.5 \mathrm{~mL}$ & $0.38 \mathrm{~mL}$ \\
\hline $\begin{array}{c}\text { CGC } \\
(91 \mathrm{~nm})\end{array}$ & $1.5 \mathrm{~mL}$ & $0.5 \mathrm{~mL}$ & $10 \mu \mathrm{L}$ & $110 \mu \mathrm{L}$ & $1 \mu \mathrm{L}$ & I & $1.5 \mathrm{~mL}$ & $0.38 \mathrm{~mL}$ \\
\hline $\begin{array}{c}\text { CGB } \\
(\mathrm{L} 72 \times \mathrm{W} 38 \mathrm{~nm})\end{array}$ & $1.5 \mathrm{~mL}$ & $0.5 \mathrm{~mL}$ & $10 \mu \mathrm{L}$ & $110 \mu \mathrm{L}$ & I & $20 \mu \mathrm{L}$ & $1.5 \mathrm{~mL}$ & $0.36 \mathrm{~mL}$ \\
\hline $\begin{array}{c}\text { CGB } \\
(\mathrm{L} 86 \times \mathrm{W} 49 \mathrm{~nm})\end{array}$ & $1.5 \mathrm{~mL}$ & $0.5 \mathrm{~mL}$ & $10 \mu \mathrm{L}$ & $110 \mu \mathrm{L}$ & I & $10 \mu \mathrm{L}$ & $1.5 \mathrm{~mL}$ & $0.37 \mathrm{~mL}$ \\
\hline $\begin{array}{c}\text { CGB } \\
(\mathrm{L} 96 \times \mathrm{W} 63 \mathrm{~nm})\end{array}$ & $1.5 \mathrm{~mL}$ & $0.5 \mathrm{~mL}$ & $10 \mu \mathrm{L}$ & $110 \mu \mathrm{L}$ & I & $5 \mu \mathrm{L}$ & $1.5 \mathrm{~mL}$ & $0.38 \mathrm{~mL}$ \\
\hline
\end{tabular}

${ }^{*} \mathrm{CGC}=$ concave gold cube; $\mathrm{CGB}=$ concave gold bar. 
Table S3. Size information of samples displayed in current work.

\begin{tabular}{|c|c|c|c|}
\hline Shape & Sample & $\begin{array}{l}\text { Size } \\
(\mathbf{n m})\end{array}$ & $\begin{array}{c}\text { Standard Deviation } \\
(\mathbf{n m})\end{array}$ \\
\hline \multirow{5}{*}{ Concave Cube } & Figure 2 & 37 & 3.7 \\
\hline & Figure $4 a$ & 42 & 3.4 \\
\hline & Figure $4 b$ & 53 & 4.3 \\
\hline & Figure $4 c$ & 69 & 5.5 \\
\hline & Figure $4 d$ & 91 & 6.5 \\
\hline \multirow{6}{*}{ Concave Bar } & \multirow{2}{*}{ Figure 5a } & 72 (length) & 7.0 (length) \\
\hline & & 38 (width) & 3.9 (width) \\
\hline & \multirow{2}{*}{ Figure $5 b$} & 86 (length) & 7.0 (length) \\
\hline & & 49 (width) & 4.7 (width) \\
\hline & \multirow{2}{*}{ Figure $5 \mathrm{c}$} & 96 (length) & 7.4 (length) \\
\hline & & 63 (width) & 5.6 (width) \\
\hline
\end{tabular}




\section{Reference}

1. Sau, T. K.; Murphy, C. J., Room temperature, high-yield synthesis of multiple shapes of gold nanoparticles in aqueous solution. J. Am. Chem. Soc. 2004, 126 (28), 8648-8649.

2. Zhang, J.; Langille, M. R.; Personick, M. L.; Zhang, K.; Li, S.; Mirkin, C. A., Concave Cubic Gold Nanocrystals with High-Index Facets. J. Am. Chem. Soc. 2010, 132 (40), $14012-14014$.

3. Francis, S.; Nuwad, J.; Gupta, A.; Sainis, J. K.; Tewari, R.; Mitra, D.; Varshney, L., Sunlight mediated synthesis of PDDA protected concave gold nanoplates. J. Nanopar. Res. 2013, 15 (3), 1482.

4. Kang, X.; Ruan, Q.; Zhang, H.; Bao, F.; Guo, J.; Tang, M.; Cheng, S.; Wang, J., Concave gold bipyramids bound with multiple high-index facets: improved Raman and catalytic activities. Nanoscale 2017, 9 (18), 5879-5886.

5. Zhang, L.-F.; Zhang, C.-Y., Controlled growth of concave gold nanobars with high surface-enhanced Raman-scattering and excellent catalytic activities. Nanoscale 2013, 5 (13), 5794-5800.

6. Huang, Y.; Wu, L.; Chen, X.; Bai, P.; Kim, D.-H., Synthesis of Anisotropic Concave Gold Nanocuboids with Distinctive Plasmonic Properties. Chem. Mater. 2013, 25 (12), 2470-2475.

7. Li, L.; Peng, Y.; Yue, Y.; Hu, Y.; Liang, X.; Yin, P.; Guo, L., Synthesis of concave gold nanocuboids with high-index facets and their enhanced catalytic activity. Chem. Commun. 2015, 
$51(58), 11591-11594$.

8. Zhang, Q.; Han, L.; Jing, H.; Blom, D. A.; Lin, Y.; Xin, H. L.; Wang, H., Facet Control of Gold Nanorods. ACS Nano 2016, 10 (2), 2960-2974.

9. Zhang, Q.; Zhou, Y.; Villarreal, E.; Lin, Y.; Zou, S.; Wang, H., Faceted Gold Nanorods: Nanocuboids, Convex Nanocuboids, and Concave Nanocuboids. Nano Lett. 2015, 15 (6), 4161-4169.

10. Rajendra, R.; Roy, D.; Tripathi, S.; Ballav, N., Facile Synthesis of Concave Cuboid Au NCs with Precisely Tunable Dimensions and Mechanistic Insight. Langmuir 2019, 35 (29), 9456-9463.

11. Thambi, V.; Kar, A.; Ghosh, P.; Paital, D.; Gautam, A. R. S.; Khatua, S., Synthesis of Complex Nanoparticle Geometries via pH-Controlled Overgrowth of Gold Nanorods. ACS Omega 2019, 4 (9), 13733-13739. 